\title{
ARTICLE
}

\section{Radiological Risk Assessment for High Dose Rate Brachytherapy with Expert Judgment and Statistical Inference}

\author{
Hyung-Joon RYU ${ }^{1}$, Han-Ki JANG ${ }^{1}$, Joo-Yeon KIM ${ }^{1}$, Kyo-Min LEE ${ }^{1}$, Jai-Ki LEE ${ }^{*}$, and Kun-Woo CHO ${ }^{2}$ \\ ${ }^{1}$ Hanyang University, 17, Haengdang-dong, Seongdong-gu, Seoul 133-791, Korea \\ ${ }^{2}$ Korea Institute of Nuclear Safety, 34, Gwahak-ro, Yuseong-gu, Daejeon, 305-338, Korea
}

\begin{abstract}
A probabilistic risk assessment was performed for the workers in and the other persons around the HDR brachytherapy facilities. To overcome the scarcity of experience data for the variables affecting exposure potential, Delphi surveys were applied to complement the available data. Bayesian inference was also employed to refine the distributions of variables. The radiological risks were evaluated by using the two-diensional Monte Carlo analysis to get better insights taking into account both uncertainty and variability. By comparing the results with those of simple probabilistic risk analysis, the merit of the approach taken in this study was exemplified.
\end{abstract}

\section{KEYWORDS: HDR brachytherapy, probabilistic risk assessment, Monte-Carlo analysis, expert judgment,} Bayesian inference

\section{Introduction}

Around 1,400 patients by year are treated with the remote afterloader brachytherapy equipments installed nationwide in Korea. ${ }^{1)}$ Most of the afterloaders used in these days are high dose rate (HDR) system which contains a sealed source of ${ }^{192}$ Ir between 100 to $400 \mathrm{GBq}$ connected to the driving wire. Due to the facts that the afterloading machine is portable, the source activity is high, the source is moving within the narrow guide tubes and catheters by a motor-driven control system and the source should be changed often because of the short halflife(74 days), the system is somewhat prone to incidents or accidents. The International Atomic Energy Agency (IAEA) and the International Commission on Radiological Protection (ICRP) have addressed this issue of potential risk in facilities practicing HDR brachytherapy ${ }^{2,3)}$.

We attempted in this study to assess the risk of medical staff members involving HDR brachytherapy and other persons potentially at risk of exposure around the facility in Korea. An event tree was constructed to set the exposure scenarios and the dose calculation models.

The values of variables used in the dose model however are subject to considerable uncertainty due to scarcity of practical data. For entire procedure of HDR brachytherapy, over 500 accidents have been reported from worldwide ${ }^{5)}$ although the majorities are relevant to patient exposure. Unfortunately the reported incident data are very rare in Korea. To complement this deficiency, Delphi surveys were conducted to draw expert judgment. In addition to the conventional Monte Carlo analysis (1D MCA), the two dimensional Monte Carlo analysis (2D MCA) was employed in the risk calculations to quantify uncertainties associated with the estimates. In addition, attempts were made to improve quality of input data by applying the Bayesian updates techniques.

*Corresponding Author, E-mail:jakilee@hanyang.ac.kr Tel.: +82-2-2220-0466, Fax: +82-2-2296-3690

\section{Materials and Method}

Generally, risk is evaluated with the products of the frequency and consequence associated with possible states of a system and with the summation of all risk values for various states of system as follows ${ }^{4)}$

$$
\text { Risk }=\sum_{i}\left(\text { Frequency }_{i} \times \text { Consequences }_{i}\right)
$$

where $i$ presents the number of possible states.

The frequency is product of the total numbers of task by the probability of the state and the consequences are calculated by use of the exposure models relevant to the states. The dose models used in this study largely follow those models developed by US Nuclear Regulatory Commission $(\mathrm{NRC})^{4)}$ under the assumption that mode of exposures should be similar although the variables affecting doses may differ due to different environmental or cultural aspects.

US NRC presented the basic scenario of risk assessment as the event tree analysis made by either success or failure of safety functions composed with confinement, shielding and access. We just considered the task of 'patient treatment' of the procedures of HDR brachytherapy. The 'patient treatment' is defined as all the tasks done in the treatment room directly related to the therapy procedures. Hence, patient treatment includes preparing patient, treatment setup and arrangement at post-treatment as well as treatment itself. In this study, a new set of safety functions consisting of source transfer, automatic source extraction, areal monitoring and access control was modeled particularly for the patient treatment to make the exposure scenarios more realistic.

To set the values of input variables, surveys with questionnaires were made in order to complement the insufficient field data. To develop questionnaires, we analyzed the task of HDR brachytherapy and set the safety factors needed to make an event scenario, and then 
constructed an event tree for possible states based on the success or the failure of safety factors. The panel of experts consists of two groups: one of 9 technologists the other of 11 medical physicists. For simple variables for which the first results are fairly consistent among the panel members, those results were taken as the final distribution. Otherwise the typical procedure of Delphi study ${ }^{6,7)}$ followed. The surveys were conducted through e-mails. The discrete data for input variables determined from Delphi study were converted into probability distribution functions(PDFs) using the Batch-fit function in Crystalball.

The probabilistic risk assessments were performed for the worker involving the treatments and other persons around the facility. The event tree includes both normal and accidental exposure situations. The 2D MCA takes into account the uncertainty and the variability of the variables in the risk model. While the 1D MCA provides a single distribution of the output, the 2D MCA generates multiple distributions so that, for example, a confidence interval for the $95 \%$ ile can be constructed from the results. The number of output distributions depends on the number of outer loop calculations. In this study, the number of inner and outer loops was 100000 and 250 , respectively.

The radiological risk can be calculated by the products of the annual frequency of treatment patient and the doses calculated by using exposure model with variables sampled from the PDFs derived from expert judgment.

One of the debates on Delphi method is how to insure the validity when experts' subjectivities could be to some degree reflected in Delphi survey. In order to complement the validity of Delphi method, parameter update using Bayes' theorem was tried out by setting the results of the first and the second Delphi surveys as the prior and likelihood, respectively. WinBUGS(V.1.4.3) which is the statistical package based on the Markov Chain Monte Carlo and Gibbs sampling method ${ }^{8)}$ is used to calculate the posterior of input variables. The results with Bayesian update were compared with those without the update.

\section{Results and Discussion}

Fig. 1 Failure probabilities of safety functions derived from Delphi panels of medical physicists(M.P) and technicians.

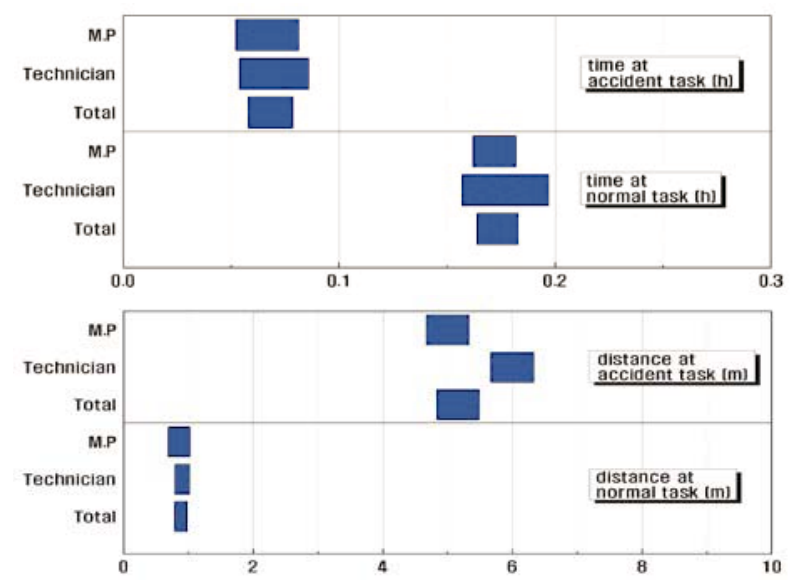

Fig. 2 Values of input variables for exposure model from $3^{\text {rd }}$ Delphi survey in normal and accident conditions.

Figure 1 shows the failure probabilities of the safety functions obtained through the Delphi surveys and their feedbacks from each expert group. Some of the variables to be used in calculation of doses were also quantified in the same way and the results for the exposed time and the distance from the source are shown in Fig. 2. Though there are some differences between the results of medical physicist(M.P) and radiological technician panels, the results are close enough to determine appropriate PDFs for the input variables.

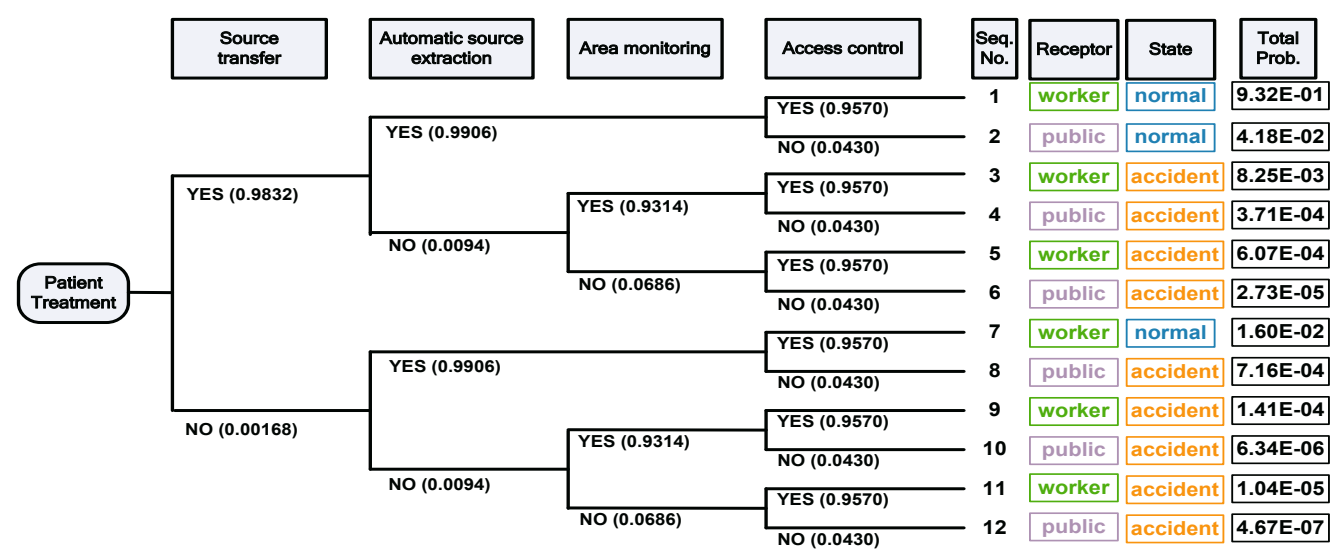

Fig. 3 Event tree for treatment task of HDR brachytherapy 
The results of event tree applying failure probabilities of each safety functions obtained from Delphi surveys are shown in Fig. 3. Exposure group was categorized as worker and public whether access control is success or not. The state whose total probability is greater than 0.01 is regarded as a normal state for the purpose of this study, otherwise an accident state. If the source does not extract automatically at treatment aborted, workers should enter the treatment room and retract the source manually. Hence, this event causes accident states.

The risks obtained from 1D MCA for the worker and the public in normal and accident situations are shown in Fig. 4 as the cumulative distribution functions (CDFs).

For identifying whether sample values from the lower- and higher-tail of the distribution make a bias to the risk results or not, we calculated the risks by rejecting the samples outside the $95 \%$ confidence interval, and compared with the results using third Delphi surveys and those using Bayesian updates.
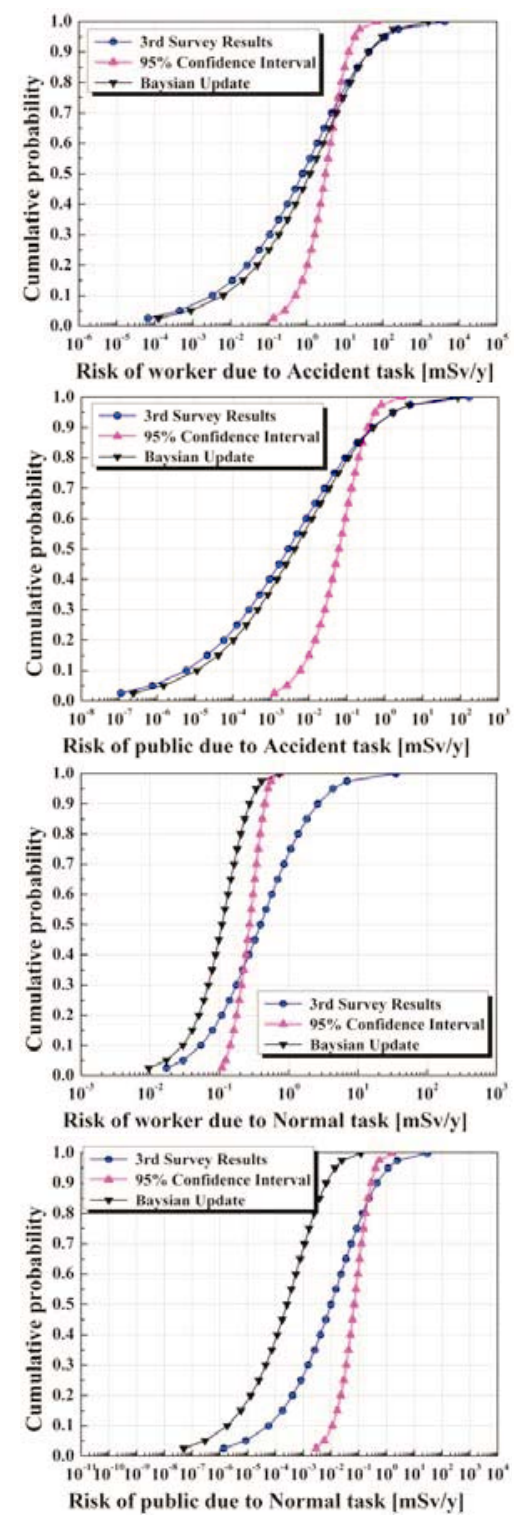

Fig. 4 1D MCA results of risk by receptors for normal and accident tasks.
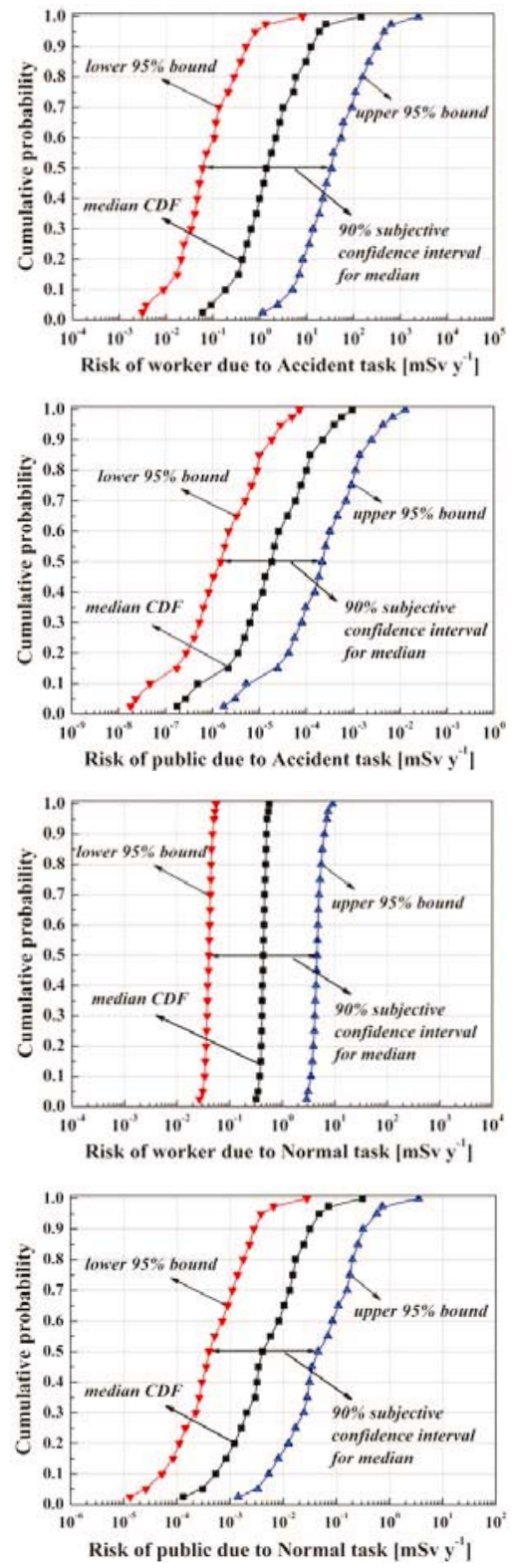

Fig. 5 2D MCA results of risk by receptors for normal and accident tasks. The subjective $90 \%$ confidence intervals for the medians are marked for examples.

The kurtosis of probabilistic distribution is quite high at the risks using samples within the $95 \%$ confidence interval. It means that there are certain variables whose values are biased toward high safety, thus resulting lower doses. Such a bias can be caused by the different background of the experts like working environment, procedures and practices as well as the personal experience. For similar reason, the probabilistic distributions have larger deviations for the accident situation than the normal one because objective data on accidents are rare so the judgment is prone to bias. Hence, in order to improve validity of the results, updating the variables from the first Delphi surveys by Bayesian method is of worth to try in the risk assessment for very uncertain situations.

The 2D MCA results provide more information than that of 1D MCA. Figure 5 shows part of the 2D MCA results of 
risks for the worker and the public. It shows two CDF curves each of which presenting the upper and lower $95 \%$ bound, respectively, and from which the $90 \%$ subjective confidence intervals for a given percentile can be deduced. By
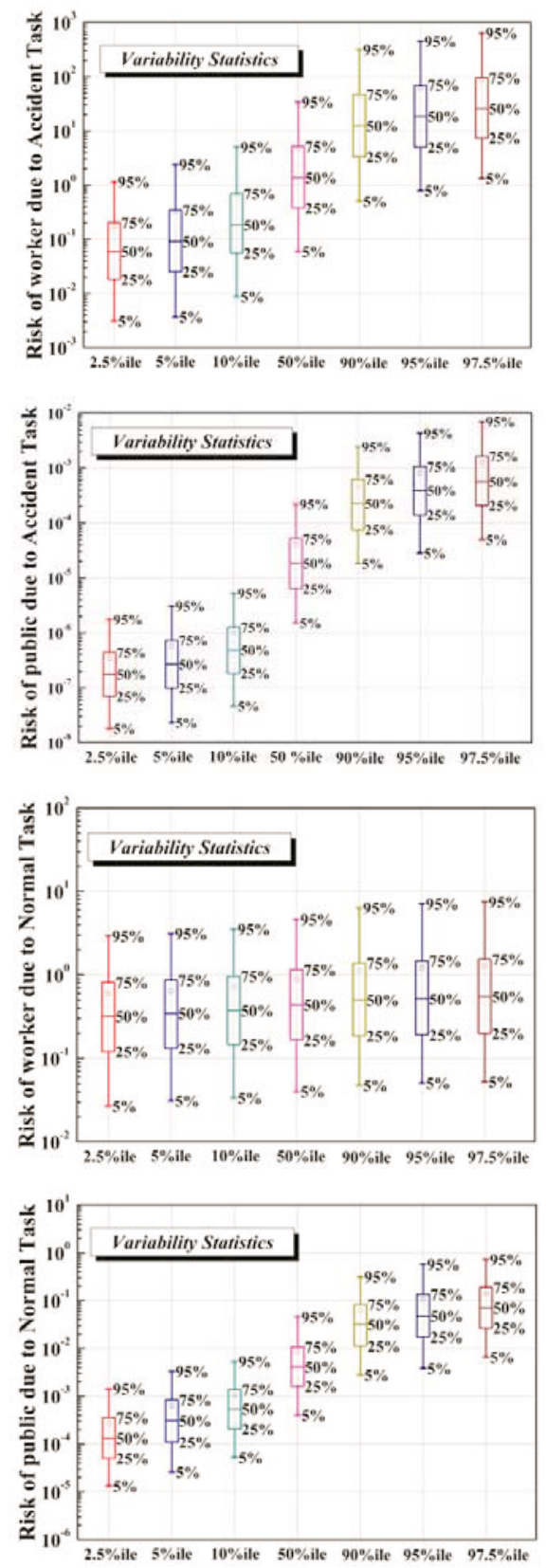

Fig. 6 2D MCA results of risks by receptor and state reflecting variability.

summarizing the statistics, the variability of risks for the worker and the public is presented in Fig. 6.

In Table 1, the risks obtained through this study are compared with those of US NRC for the task of patient treatment in HDR brachytheraphy. While US NRC presented single values about risk, the results of this study present 5 and 95 percentiles of the median. The estimated risks in terms of annual doses roughly agree with each other for different cases. It is noted that in our results, except for the
Table. 1 Comparison between risks for treatment task assessed by US NRC and this study(2D MCA)

\begin{tabular}{|c|c|c|c|c|}
\hline \multirow{3}{*}{ Risk } & \multicolumn{2}{|c|}{$\begin{array}{c}\text { US NRC } \\
\text { (medians) }\end{array}$} & \multicolumn{2}{|c|}{$\begin{array}{c}\text { This study } \\
(90 \% \text { confidence intervals } \\
\text { for the median) }\end{array}$} \\
\cline { 2 - 5 } & Normal & Accident & Normal & Accident \\
\hline Worker & $1.47 \times 10^{-1}$ & $5.43 \times 10^{-2}$ & $\begin{array}{c}{[0.189,} \\
0.737]\end{array}$ & $\begin{array}{c}{\left[2.96 \times 10^{-4}\right.} \\
\left.2.78 \times 10^{2}\right]\end{array}$ \\
\hline Public & 0.00 & $8.40 \times 10^{-2}$ & $\begin{array}{c}{\left[5.43 \times 10^{-6},\right.} \\
2.15]\end{array}$ & $\begin{array}{c}{\left[9.54 \times 10^{-8}\right.} \\
7.63]\end{array}$ \\
\hline
\end{tabular}

workers at normal task, the confidence intervals are rather wide. Therefore any single estimate of risk does not give full insight of the risk underlies particularly for systems where highly uncertain variables are involved. This fact exemplifies the utility of 2D MCA in risk analysis.

\section{Conclusion}

Radiological risks associated with the treatment procedures in HDR brachytherapy were assessed probabilistically by applying 2D MCA combined with Bayesian update of expert judgment on the variables affecting the risk. It is found that the risk information for the treatment task is improved by providing more meaningful results compared to those of 1D MCA or simple probabilistic risk analysis. Although the scope of analysis in this study is limited to a narrow task, treatment itself, the methodology tried out in this study can be applied to more complicated systems.

\section{Acknowledgment}

This work was supported by Korean Ministry of Knowledge Economy (2008-P-EP-HM-E-06-0000), Ministry of Education, Science and Technology of Korea, and Sunkwang Atomic Energy Safety Co., Ltd..

\section{References}

1) Y. Ji, M. Kim, S. Ryu, D. Yu, M. Choi, H. Jeong, National statistics of radiation oncology in Korea (in 2006), J Korean Soc Ther Radiol Oncol, 26(2) (2008) 131-133

2) ICRP, Radiological protection and safety in medicine, ICRP publication 73, (1996).

3) IAEA, Generic procedures for assessment and response during a radiological emergency, IAEA-TECDOC-1162, (2000).

4) U.S. Nuclear Regulatory Commission, Risk analysis and evaluation of regulatory options for nuclear byproduct material systems, NUREG/CR-6642, (2000).

5) ICRP, Prevention of High-Dose-Rate brachytherapy accidents, ICRP publication 97, (2004)

6) M. A. Meyer, J. M. Booker, Eliciting and analyzing expert judgment, American Statistical Association, (2001)

7) A.O'Hagan, C. E. Buck, A. Daneshkhah, J. R. Eiser, P. H. Garhwaite, D. J. Jenkinson, J. E. Oakley, T. Rakow, Uncertain judgements: Eliciting experts' probabilities, John Wiley \& Sons, Ltd., (2006)

8) http://www.mrc-bsu.cam.ac.uk/bugs, D. Spiegelhalter, A. Thomas, N. Best, D. Lunn, WinBUGS User Manual, (2003) 